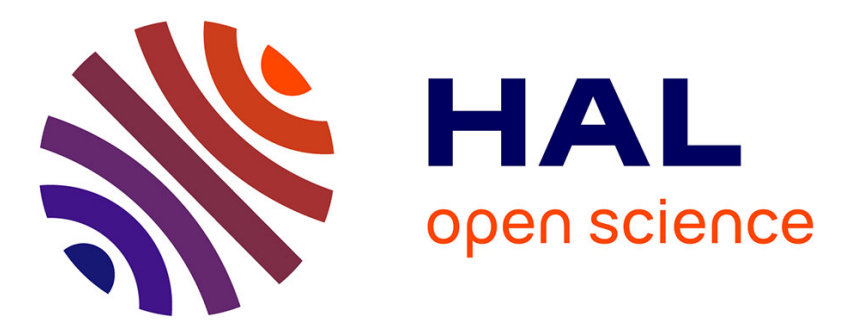

\title{
Propriétés macroscopiques équivalentes pour représenter les pertes dans les bobines conductrices
}

Gérard Meunier, Anh Tuan Phung, Olivier Chadebec, Xavier Margueron, Jean-Pierre Keradec

\section{- To cite this version:}

Gérard Meunier, Anh Tuan Phung, Olivier Chadebec, Xavier Margueron, Jean-Pierre Keradec. Propriétés macroscopiques équivalentes pour représenter les pertes dans les bobines conductrices. Revue Internationale de Génie Electrique, 2008, 11 (6), pp.675-694. 10.3166/rige.11.675-694 hal-00360724

\section{HAL Id: hal-00360724 https://hal.science/hal-00360724}

Submitted on 11 Feb 2009

HAL is a multi-disciplinary open access archive for the deposit and dissemination of scientific research documents, whether they are published or not. The documents may come from teaching and research institutions in France or abroad, or from public or private research centers.
L'archive ouverte pluridisciplinaire $\mathbf{H A L}$, est destinée au dépôt et à la diffusion de documents scientifiques de niveau recherche, publiés ou non, émanant des établissements d'enseignement et de recherche français ou étrangers, des laboratoires publics ou privés. 


\title{
Propriétés macroscopiques équivalentes pour représenter les pertes dans les bobines conductrices
}

\author{
Gérard Meunier - Anh-Tuan Phung - Olivier Chadebec - Xavier \\ Margueron - Jean-Pierre Keradec \\ Laboratoire de Génie Electrique de Grenoble - G2Elab \\ UMR 5269 INPG-UJF-CNRS \\ ENSIEG - BP46 \\ F-38402 Saint-Martin-d'Hères Cedex \\ gerard.meunier@leg.ensieg.inpg.fr
}

RÉSUMÉ. La prise en compte des pertes dans les bobinages comprenant un grand nombre de conducteurs est rapidement hors de portée des logiciels par éléments finis en trois dimensions. Nous proposons comme alternative une technique d'homogénéisation par l'intermédiaire de propriétés macroscopiques complexe. Un exemple test valide la démarche proposée.

ABSTRACT. The calculation of eddy current losses in the windings, which have a big number of conductors, is very quickly out of FEM software capacity in 3D. We introduce one alternative technique by using macroscopic complex properties. A test case will be presented to validate the proposed approach.

MOTS-CLÉS : haute fréquence, conducteurs rectangulaires, pertes par effet de proximité, perméabilité complexe, méthode des éléments finis,

KEYWORDS: high frequency, rectangular conductors, proximity losses, complex permeability, finite element method.

Revue. Volume $\mathrm{X}-\mathrm{n}^{\circ} \mathrm{x} /$ année, pages 1 à $\mathrm{X}$ 


\section{Introduction}

Les pertes dynamiques dans les bobinages des transformateurs sont difficiles à modéliser avec la méthode des éléments finis en raison du très grand nombre de brins conducteurs composant les bobinages. Le problème est particulièrement crucial en trois dimensions, car la détermination des courants de Foucault nécessite une inconnue vectorielle dans les milieux conducteurs. Avec la montée en fréquence, l'épaisseur de peau devient rapidement petite par rapport aux dimensions des conducteurs. Nous savons par ailleurs que pour obtenir une modélisation précise avec la méthode des éléments finis, il est préférable de disposer au moins deux mailles dans l'épaisseur de peau. Cette condition génère un maillage très souvent incompatible avec les moyens de calcul disponibles, particulièrement en 3 dimensions. Pour ces raisons, nous allons rechercher des méthodes alternatives pour prendre en compte ces pertes.

Les pertes par courants de Foucault dans les brins conducteurs d'une bobine ont pour origine le champ magnétique variable agissant sur un milieu massif conducteur : en première approximation ces pertes peuvent se décomposer suivant deux origines: les pertes propres dues à l'effet de peau par le biais du champ magnétique créé par le conducteur sur lui-même et celles dues aux effets de proximité par le biais d'un champ extérieur. Ce dernier a lui-même pour origine des bobines extérieures mais aussi le champ créé par les autres brins composant la bobine elle-même.

Cet article a comme objectif de compléter les travaux existants sur la représentation des pertes dans les bobinages (Phung et al., 2007), (Gyselinck at al., 2005) (Nan et al., 2004) (Matagne, 1995), (Moreau et al., 1998) (Ferreira, 1994) et de montrer comment les pertes locales au niveau des brins conducteurs peuvent être traduites par des propriétés équivalentes (perméabilité, conductivité) au niveau macroscopique. Nous préciserons comment obtenir ces grandeurs en régime harmonique, à partir de la résolution locale des équations de champ au niveau des brins conducteurs. Dans le cas de conducteurs ronds, carrés ou rectangulaires des solutions analytiques permettent de calculer ces propriétés : les techniques proposées, sont performantes et faciles à mettre en œuvre. Elles s'avèrent cependant relativement imprécises pour les conducteurs rectangulaires en haute fréquence. Pour remédier à cette difficulté, une méthode numérique générale, basée sur une technique d'homogénéisation est proposée dans cet article. Elle donne des résultats très satisfaisants lors des évaluations de pertes en 2D. Un exemple 3D permet d'étendre et de valider la démarche proposée.

Nous plaçons notre étude à une fréquence suffisamment faible pour pouvoir résoudre les équations de Maxwell sans prendre en compte les courants de déplacement. Dans ces conditions, les champs recherchés sont l'excitation $\mathrm{H}$ et l'induction magnétique $\mathrm{B}$ dans tout de domaine d'étude, associés à la densité de courant $\mathrm{J}$ et au champ électrique $\mathrm{E}$ dans les milieux conducteurs. Les lois de comportement associées relient $\mathrm{H}$ et $\mathrm{B}$ d'une part et $\mathrm{J}$ et $\mathrm{E}$ d'autre part. 
2. Considérations générales sur les pertes dans une bobine

Nous considérons le cas d'une bobine filaire, comprenant un grand nombre de brins et où chaque brin est parcouru par un courant identique. A l'échelle macroscopique nous résolvons, avec la méthode des éléments finis, les équations de champ suivantes:

$$
\begin{aligned}
\operatorname{rot} \mathbf{H} & =\mathbf{J} \\
\operatorname{div} \mathbf{B} & =0
\end{aligned}
$$

en considérant $\mathbf{J}$ comme étant uniforme du fait de la taille des brins élémentaire par rapport aux dimensions macroscopiques. Nous ne résolvons pas localement l'équation de Maxwell-Faraday, mais écrivons la densité de courant macroscopique $\mathrm{J}$ sous la forme:

$$
\mathrm{J}=\mathrm{nI}
$$

En définissant $\mathrm{n}$ comme la densité spires et I le courant traversant une spire. En supposant que les courants de Foucault sont négligeables, seules les pertes ohmiques statiques interviennent et la relation qui relie le courant à la tension de la spire s'écrit, en utilisant le potentiel vecteur magnétique A:

$$
\mathrm{U}=\mathrm{R} . \mathrm{I}+\mathrm{j} \omega \int_{\Omega_{\mathrm{bob}}} \mathbf{n} . \mathbf{A} \mathrm{d} \Omega
$$

$$
\text { avec } R=\frac{\rho}{\lambda} n^{2} V_{\text {bob }}=\rho_{\text {eqs }} n^{2} V_{\text {bob }}
$$

où $\lambda$ représente le coefficient de foisonnement, $\rho$ la résistivité du conducteur, $\rho_{\text {eqs }}$ la résistivité équivalente statique et $\mathrm{V}_{\text {bob }}$ le volume de la bobine. La puissance magnétique sur la bobine s'écrit:

$$
\begin{aligned}
\mathrm{P} & =\mathrm{U.I}^{*}=\int_{\Omega_{\mathrm{bob}}} \mathbf{J} \mathbf{E}^{*} \mathbf{d} \Omega+\mathrm{j} \omega \int_{\Omega_{\mathrm{bob}}} \mathbf{B} \mathbf{H}^{*} \mathbf{d} \Omega \\
& =\text { R.I }{ }^{2}+\mathrm{j} \omega \int_{\Omega_{\mathrm{bob}}} \mu_{\mathbf{o}} \mathbf{H}^{2} \mathrm{~d} \Omega
\end{aligned}
$$

En pratique, des courants locaux se développent à l'échelle du brin et contribuent à augmenter les pertes, modifiant la tension et la puissance dissipée dans la bobine.

Nous plaçons notre étude suivant deux échelles : l'échelle macroscopique où nous souhaitons décrire les bobines en homogénéisant les brins conducteurs suivant des propriétés équivalentes et l'échelle des brins conducteurs, que nous appellerons échelle locale. Nous supposons que les dimensions macroscopiques (celles du dispositif que nous souhaitons simuler) sont beaucoup plus grandes que la taille des brins conducteurs. Dans un premier temps, nous allons montrer de façon qualitative, que les pertes supplémentaires dans les bobines, qui ont pour origine 
l'interaction du champ magnétique avec les brins conducteurs, peuvent se traduire, au niveau macroscopique, par des lois de comportement modifiées par rapport au cas statique.

Les équations de Maxwell sont vérifiées aux deux échelles, par les champs locaux et macroscopiques. A l'échelle locale, les lois de comportement reliant les champs locaux e, b, h, j sont connues. Nous avons dans le cas d'une bobine:

$\mathrm{b}=\mu_{0} \mathrm{~h}$

$\mathrm{j}=\sigma \mathrm{e}$ (dans les brins conducteurs uniquement)

où $\sigma$ est la conductivité électrique. Les équations résolues localement sont:

$$
\begin{array}{ll}
\operatorname{rot} \mathbf{h}=\mathbf{j} & \operatorname{div} \mathbf{b}=0 \\
\operatorname{rot} \mathbf{e}=-\frac{\partial \mathbf{b}}{\partial \mathbf{t}} & \operatorname{div} \mathbf{j}=0
\end{array}
$$

Nous cherchons, à partir de la résolution de ces équations et de la connaissance de lois locales précédentes, à établir les lois de comportement à l'échelle macroscopique reliant les grandeurs E, B, H, J. Pour cela, nous définissons les différences des champs locaux avec les grandeurs macroscopiques suivant:

$$
\begin{array}{ll}
\mathbf{h}=\mathbf{H}+\mathbf{h}_{\mu} & \mathbf{b}=\mathbf{B}+\mathbf{b}_{\mu} \\
\mathbf{e}=\mathbf{E}+\mathbf{e}_{\mu} & \mathbf{j}=\mathbf{J}+\mathbf{j}_{\mu}
\end{array}
$$

Avec les définitions données en [5], il est important de remarquer deux points :

- En premier lieu la conservation des flux (Maxwell-Gauss) et des courants, qui exprime que le flux moyen à travers une surface est l'intégrale de la grandeur locale, conduit à écrire :

$$
\begin{aligned}
& B=b \mid \\
& J=\mid j
\end{aligned}
$$

où $\langle\mathrm{b}\rangle$ est défini comme la valeur moyenne volumique de $\mathrm{b}$ (en d'autres termes $\left\langle\mathrm{b}_{\mu}\right\rangle=0$ et $\left\langle\mathrm{j}_{\mu}\right\rangle=0$ ce qui signifie que $b_{\mu}$ et $\mathrm{j}_{\mu}$ se présentent sous la forme de boucles de champ et de courant).

- En second lieu nous allons montrer que cette propriété n'est pas nécessairement vérifiée pour h et e en régime dynamique. Prenons le cas du champ magnétique $\mathrm{H}$ : ce dernier doit vérifier le théorème d'Ampère (Maxwell-Ampère) au niveau macroscopique. Dans l'hypothèse où les courant de boucle créent un champ moyen $\left\langle\mathrm{h}_{\mu}\right\rangle$ différent de zéro la relation $\operatorname{rot}(\langle\mathrm{h}\rangle)=\langle\mathrm{j}\rangle$ est incorrecte dans la mesure où la densité de courant macroscopique $\mathrm{J}=\langle\mathrm{j}\rangle$ ne voit pas les courants de boucle $\mathrm{j}_{\mu}$ et que le champ moyen $\langle\mathrm{h}\rangle$ donne une contribution qui inclue les boucles de courants produites par $\mathrm{j}_{\mu}$. En d'autres termes, au niveau macroscopique, $\mathrm{H}$ ne voit que les courants consolidés et ignore le champ créé par les boucles 
locales. Nous devons donc prendre en compte, pour la contribution au champ $\mathrm{H}$, les champs $\left\langle\mathrm{h}_{\mu}\right\rangle$ créés par les courants de boucle et écrire pour $\langle\mathrm{h}\rangle$ :

$$
<\mathrm{h}>=\mathrm{H}+<\mathrm{h}_{\mu}>
$$

Un raisonnement analogue peut être mené avec le champ électrique e et l'équation de Maxwell-Faraday, en utilisant les boucles de champ. Nous écrivons

$$
<\mathrm{e}>=\mathrm{E}+<\mathrm{e}_{\mu}>
$$

Nous allons mettre en évidence ce phénomène dans notre cas suivant deux configurations: un brin conducteur soumis à un champ extérieur et un brin conducteur sous l'action de son propre champ (Robert, 1999).

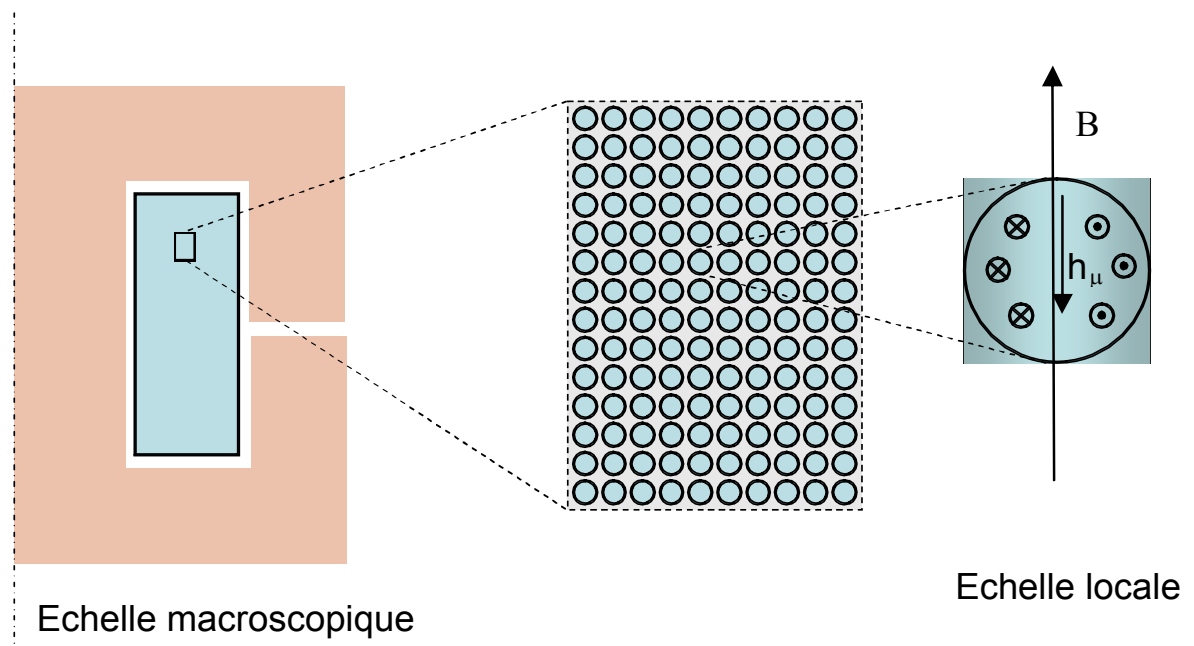

Fig. 1 Courants induits par un champ B sur un brin d'une bobine (effet de proximité) : les courants locaux dans le brins créent un champ $\mathbf{h}_{\mu}$ qui s'oppose à $B$

Considérons une bobine, composée d'un grand nombre de brins conducteurs. Chaque brin est soumis à un champ extérieur provenant d'autres bobines ou des autres brins de la même bobine. La figue 1 montre l'allure des courants induits locaux engendrés par ce champ extérieur. Puisque les brins sont isolés les uns par rapport aux autres, ces courants ne peuvent pas se consolider au niveau macroscopique et restent sous forme de boucles à l'échelle locale.

Suivant la loi de Lenz, les boucles de courants créées s'opposent au champ inducteur et sont toutes orientées dans un sens opposé à B. Elles contribuent ainsi à la création d'un champ moyen macroscopique non nul $\left\langle\mathrm{h}_{\mu}\right\rangle$ qui s'oppose à $\mathrm{dB} / \mathrm{dt}$ (et qui est fonction de $\mathrm{dB} / \mathrm{dt}$ ). Nous avons, compte tenu que $\mathrm{b}=\mu_{\mathrm{o}} \mathrm{h}$ au niveau local : 
$\mathrm{B}=\langle\mathrm{b}\rangle=\mu_{\mathrm{o}}\langle\mathrm{h}\rangle=\mu_{\mathrm{o}}\left(\mathrm{H}+\left\langle\mathrm{h}_{\mu}\right\rangle\right)$

Cela nous suggère que la loi de comportement macroscopique qui relie $\mathrm{B}$ à $\mathrm{H}$ au niveau macroscopique diffère de la loi statique dès que $\mathrm{dB} / \mathrm{dt}$ est différent de zéro. On peut interpréter cela en considérant que les boucles de courant agissent comme des dipôles magnétiques contribuant à une aimantation moyenne $\left\langle\mathrm{h}_{\mu}\right\rangle$ au niveau macroscopique. En régime harmonique, nous pourrons traduire ce phénomène par l'intermédiaire d'une perméabilité complexe fonction de la fréquence.

Considérons maintenant le cas d'un brin conducteur soumis à son propre champ, créé par le courant (variable dans le temps) le traversant. La figure 2 montre l'allure des boucles de courants induits et des lignes de champ engendrées dans cette situation (effet de peau classique).

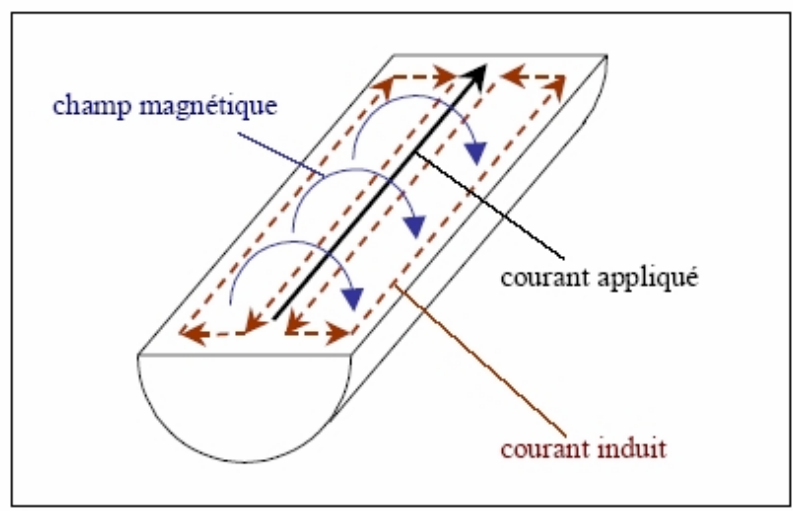

Fig. 2 Courants induits par le courant du conducteur (effet pelliculaire)

Nous pouvons constater, dans ce cas, que les boucles de courants $j_{\mu}$ engendrent un champ magnétique dont la valeur moyenne $\left\langle\mathrm{h}_{\mu}\right\rangle$ est nulle. En pratique, ces courants n'engendrent pas de modification de la loi magnétique macroscopique. A contrario, nous sommes en présence d'une situation où le champ électrique local moyen $\left\langle\mathrm{e}_{\mu}\right\rangle$ est différent de zéro. En effet nous pouvons constater que les boucles de courants $\left\langle\mathrm{j}_{\mu}\right\rangle$ sont associées à des boucles de champ qui traduisent la présence d'un champ électrique $e_{\mu}$ dont la valeur moyenne est non nulle. Cela conduit à une modification de la loi électrique suivant (en tenant compte du coefficient de foisonnement $\lambda$ ):

$$
\mathrm{J}=<\mathrm{j}>=\lambda<\sigma \mathrm{e}>=\lambda \sigma\left(\mathrm{E}+\mathrm{e}_{\mu}>\right)
$$

Les boucles de champ agissent comme des dipôles électriques et contribuent à modifier la loi de comportement macroscopique $J(E)$. En régime harmonique nous pourrons traduire ces phénomènes sous la forme d'une conductivité complexe dont les parties réelles et imaginaires varient avec la fréquence. 
En pratique, les deux phénomènes co-existent (Fig. 3). Dans la suite nous allons présenter comment calculer ces lois de comportement macroscopiques pour les bobines par des méthodes numériques, après avoir abordé succinctement la possibilité d'utiliser des méthodes analytiques.

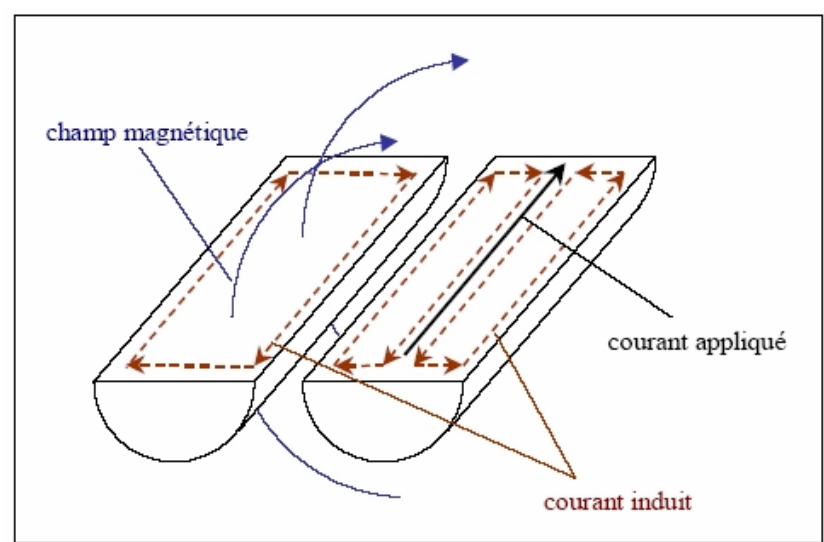

Fig. 3 Effet propre et effet de proximité créé par un conducteur alimenté

\section{Méthodes analytiques}

\subsection{Conducteurs ronds}

De nombreux travaux existent sur ce sujet où le principe général consiste à déterminer une perméabilité complexe équivalente lorsque qu'un brin est soumis à un champ extérieur uniforme, puis d'homogénéiser les brins en tenant compte de l'espacement entre chaque brin conducteur et de l'interaction entre ces derniers. Dans le cas des conducteurs ronds une solution analytique peut être établie pour la perméabilité équivalent (Matagne, 1993) mais aussi pour la conductivité équivalente (Matagne 1997), (Stoll, 1974). D'autre travaux, notamment basés sur le principe des plaques de Dowell et appliqués aux conducteurs ronds, avec une décomposition du champ appliqué suivant les effets propres et proximité ont été également développés (Ferreira, 1994).

\subsection{Conducteurs rectangulaires}

Dans le cas des conducteurs rectangulaires, la solution pour un brin peut être approchée par la superposition de deux solutions 1D qui donnent respectivement la perméabilité équivalente dans les deux directions (Moreau et al., 1998). Après homogénéisation, nous obtenons une perméabilité équivalente pour un ensemble de brins conducteurs. Si le champ de réaction d'un brin conducteur est faible par rapport au champ appliqué, ces méthodes donnent toujours de très bons résultats. 
C'est généralement le cas lorsque la fréquence est suffisamment faible. Ces méthodes trouvent toutefois leur limitation lorsque les hypothèses de détermination de la perméabilité complexe par des méthodes analytiques ne sont plus vérifiés, c'est-à-dire lorsque le champ local appliqué au niveau de chaque brin n'est pas uniforme. Or ce phénomène, qui est liée aux courants induits locaux agissant sur le brin et à l'interaction entre les brins, devient rapidement significatif lorsqu'un conducteur n'est plus rond ou carré (Moreau et al., 1998).

\subsection{Intérêts et limites des méthodes analytiques}

Les méthodes analytiques ont un grand intérêt car elles permettent d'accéder très rapidement à une représentation correcte des pertes supplémentaires pour peu que la géométrie des brins conducteurs soit suffisamment simple. Ces méthodes trouvent cependant leurs limitations dans la prise en compte exacte du problème local : il est difficile de prendre correctement en compte l'interaction entre les brins conducteurs et il n'existe pas de solution analytique pour des géométries quelconques. L'utilisation de méthodes numériques, comme celle des éléments finis que nous utilisons ci-dessous, permet de s'affranchir de ces difficultés (Nan et al., 2004).

\section{Méthodes numériques}

Nous développons dans cette partie l'apport des méthodes numériques pour le calcul des propriétés équivalentes, ces dernières permettant d'accéder aux champs locaux à travers des résolutions de type éléments finis. Nous présentons tout d'abord quelques principes généraux et développons ensuite comment évaluer les champs locaux à partir des champs macroscopiques en utilisant une technique d'homogénéisation.

\section{1 Champs macroscopiques à l'échelle locale}

Pour calculer les propriétés équivalentes à l'échelle macroscopique, nous considérons un domaine suffisamment petit pour que l'induction macroscopique $B$ et la densité de courant $\mathrm{J}$ puissent y être considérés comme étant uniformes. Ces grandeurs vont constituer les termes sources de notre problème local. Notons que les champ $\mathrm{H}$ et $\mathrm{E}$ varient linéairement sur le domaine local dès que $\mathrm{B}$ et $\mathrm{J}$ sont différents de zéro puisqu' étant reliés à $\mathrm{B}$ et $\mathrm{J}$ par les lois de Maxwell-Ampère et Maxwell-Faraday:

$$
\begin{aligned}
& \operatorname{rot} \mathbf{H}=\mathbf{J} \\
& \operatorname{rot} \mathbf{E}=-\frac{\partial \mathbf{B}}{\partial \mathrm{t}}
\end{aligned}
$$

Après résolution du problème local, nous sommes susceptibles de déterminer les valeurs de $\mathrm{H}$ et $\mathrm{E}$ sur le domaine élémentaire. Nous écrivons alors les relations 
macroscopiques $\mathrm{H}(\mathrm{B})$ et $\mathrm{J}(\mathrm{E})$ en moyennant les valeur de $\mathrm{H}$ et $\mathrm{E}$ sur le domaine élémentaire. Ainsi, dans le cas de brins conducteurs, les propriétés équivalentes en 2D (avec les courants perpendiculaires au plan d'étude), sont définies par l'intermédiaire de grandeurs complexes (tensorielle pour la reluctivité) suivant:

$$
\begin{aligned}
& <\mathrm{H}>=\left[v_{\text {eq }}\right] \mathrm{B} \\
& <\mathrm{E}>=\rho_{\text {eq }} \mathrm{J}
\end{aligned}
$$

Cette démarche, qui utilise des champs uniforme B et $\mathrm{J}$ sur le domaine élémentaire, traduit également le fait que les lois de comportement ne sont pas couplées. Ce point est développé au paragraphe 4.7.

Dans le paragraphe 4.3 nous allons montrer comment les techniques d'homogénéisation permettent d'exprimer le problème local en fonction de $\mathrm{B}$ et $\mathrm{J}$ et de retrouver les propriétés équivalentes. En pratique si nous savons déterminer les grandeurs $\left\langle\mathrm{h}_{\mu}\right\rangle$ et $\left\langle\mathrm{e}_{\mu}\right\rangle$ ou encore $\langle\mathrm{H}\rangle$ et $\langle\mathrm{E}\rangle$ nous en déduisons sans difficulté les propriétés équivalentes. En pratique, ces deux grandeurs ne sont pas toujours accessibles facilement et simultanément après résolution du problème local, alors que $\mathrm{h}, \mathrm{e}, \mathrm{b}, \mathrm{j}$ le sont plus généralement. Nous proposons, dans le paragraphe suivant ci dessous, une méthode pour retrouver les propriétés équivalentes à partir de la connaissance des champs locaux.

\section{2 Calcul des propriétés par équivalence énergétique}

Nous supposons savoir calculer les champs locaux à partir des champs macroscopiques B et $\mathbf{J}$ supposés uniformes sur un domaine local. Le principe général consiste alors à égaliser les puissances magnétiques, à partir des valeurs de champ locales et macroscopiques et en se référant à la définition des propriétés équivalentes ci dessus [12]:

$$
\begin{aligned}
\mathrm{P}_{\text {micro }} & =\mathrm{j} \omega \int_{\Omega} \mathbf{h} \overline{\mathbf{b}} \mathbf{d} \Omega+\int_{\Omega} \mathbf{j} \overline{\mathbf{e}} \mathbf{d} \Omega \\
\mathrm{P}_{\text {macro }} & =\mathrm{j} \omega \int_{\Omega} \mathbf{B} \overline{\mathbf{H}} \mathbf{d} \Omega+\int_{\Omega} \mathbf{J} \overline{\mathbf{E}} \mathbf{d} \Omega \\
& =\mathrm{j} \omega \mathbf{B}<\overline{\mathbf{H}}>+\mathbf{J}<\overline{\mathbf{E}}> \\
& =\mathrm{j} \omega \mathbf{B}\left[v_{\text {eq }}\right] \overline{\mathbf{B}} . \mathrm{V}+\mathbf{J} \rho_{\text {eq }} \overline{\mathbf{J}} . \mathrm{V}
\end{aligned}
$$

$\mathrm{V}$ représente le volume du domaine élémentaire. En pratique, nous nous plaçons suivant deux conditions de calcul: un calcul "magnétique" et un calcul "électrique".

Le calcul magnétique est réalisé en imposant une densité de courant nulle dans le domaine (ce qui équivaut dans notre cas à un courant nul dans les brins conducteurs), ces derniers étant soumis à un champ macroscopique B. Après calcul des grandeurs locales, nous établissons l'égalité des puissance actives et réactives suivant: 


$$
\mathrm{j} \omega \int_{\Omega} \mathrm{h} \overline{\mathrm{b}} \mathrm{d} \Omega+\int_{\Omega} \mathrm{j} \overline{\mathrm{e}} \mathrm{d} \Omega=\mathrm{j} \omega \mathrm{B}\left[v_{\text {eq }}\right] \overline{\mathrm{B}} . \mathrm{V}
$$

qui permet d'accéder aux parties réelles et imaginaires de la reluctivité équivalente $\left[v_{\mathrm{eq}}\right]$. Dans le cas de conducteurs de forme rectangulaires ou quelconques la perméabilité est tensorielle, et il est alors nécessaire de conduire deux calculs suivant les axes principaux de la cellule afin de déterminer le tenseur de reluctivité. Si les axes principaux ne sont pas connus trois calculs sont nécessaires : Dans un repère $x y$, les deux premiers calculs permettent de déterminer $v_{e} q_{x x}\left(\right.$ en imposant $B_{y}=0$ ) et veqy $\left(\right.$ en imposant $B_{x}=0$ ) alors que le dernier, suivant une direction quelconque, permet d'accéder à veq $\mathrm{q}_{\mathrm{xy}}$ connaissant $v_{\mathrm{eq}} \mathrm{q}_{\mathrm{xx}}$ et vequy.

Le calcul "électrique" consiste à imposer une densité de courant macroscopique J non nulle dans le domaine (dans notre cas imposer un courant I dans les brins conducteurs), tout en imposant une induction magnétique macroscopique nulle sur le domaine élémentaire. Nous pouvons remonter à la résistivité et à la résistance équivalente en écrivant:

$$
\begin{aligned}
& \mathrm{j} \omega \int_{\Omega} \mathbf{h} \overline{\mathbf{b}} \mathbf{d} \Omega+\int_{\Omega} \mathbf{j} \overline{\mathbf{e}} \mathbf{d} \Omega=\rho_{\mathbf{e q}} \mathbf{J} \overline{\mathbf{J}} . V \\
& \mathrm{R}_{\text {eq }}=\rho_{\text {eq }} \mathrm{n}^{2} \mathrm{~V}_{\mathrm{bob}}
\end{aligned}
$$

\section{3 Calcul des champs locaux par une technique d'homogénéisation}

Nous choisissons comme domaine élémentaire une cellule rectangulaire xy représentant un motif élémentaire (par exemple un brin conducteur et l'espacement avec ses voisins - voir un exemple sur la Fig. 4), avec l'hypothèse que le bobinage est réalisé de façon régulière. Si le nombre de brins est suffisamment grand, les champs macroscopiques B et $\mathbf{J}$ peuvent être considérés comme étant constant sur chaque cellule (en d'autres termes, le flux entrant sur un coté de la cellule est égal au flux sortant sur le coté opposé). Cette hypothèse va nous permettre de déterminer les propriétés macroscopiques équivalentes. Nous allons utiliser le fait que les champs locaux ont des propriétés de périodicité pour pouvoir appliquer des conditions aux limites correctes sur chaque cellule élémentaire. Les équations de Maxwell sont résolues en utilisant les lois de comportement statiques locales des matériaux.

Dans ce qui suit, nous nous plaçons en $2 \mathrm{D}$ en utilisant le potentiel vecteur magnétique $(b=\operatorname{rot} a)$. Les équations de champ au niveau local s'écrivent, compte tenu que $h=v_{0} b$ et $j=\sigma e$ :

$$
\begin{array}{ll}
\operatorname{rot}\left(v_{\mathrm{o}} \operatorname{rot} \mathbf{a}\right)=0 & \text { dans l'air } \\
\operatorname{rot}\left(v_{\mathrm{o}} \operatorname{rot} \mathbf{a}\right)+\mathrm{j} \omega \sigma \mathbf{a}=-\sigma \operatorname{gradV} V & \text { dans le conducteur }
\end{array}
$$

A ces équations, nous associons une équation de circuit qui nous permet d'imposer le courant total I traversant le conducteur, à une valeur nulle ou non : 


$$
I=\int_{\text {cond }} \mathrm{j} \mathrm{d} \Omega=\int_{\text {cond }}-\mathrm{j} \omega \sigma \mathrm{a}-\sigma \operatorname{grad} \mathrm{V} \mathrm{d} \Omega
$$

Nous souhaitons résoudre ces équations sur une cellule, en imposant un champ magnétique (nul ou non) sur cette dernière. Dans le cas où la géométrie de la cellule le permet, nous pouvons imposer directement des conditions aux limites particulières sur a (Cf paragraphe 4.4 pour le calcul sur des brins rectangulaires). Dans le cas le plus général, il est judicieux d'utiliser des conditions de périodicité. Remarquons cependant que nous ne pouvons pas imposer simplement une induction macroscopique B par condition aux limites sur a. En effet a ne posséde pas de caractère de périodicité avec une induction uniforme imposée, puisque A est reliée à $\mathrm{B}$ par $\mathrm{B}=$ rot $\mathrm{A}$. Nous pouvons cependant écrire sur la cellule élémentaire où $\mathrm{B}$ est supposé uniforme :

$$
\mathrm{A}=\mathrm{B} \wedge \mathrm{x}+\mathrm{A}_{0}
$$

où $\mathrm{A}_{0}$ est le potentiel vecteur macroscopique en $\mathrm{x}=0$ et où $\mathrm{x}$ est le vecteur de coordonnées $\{\mathrm{x}, \mathrm{y}, \mathrm{z}\}$ avec comme origine le centre de la cellule (ce choix n'a pas d'incidence sur le résultat final).

Remarque: D'une façon générale, la définition d'une référence pour le potentiel vecteur n'a pas d'incidence sur le résultat final en terme de champ magnétique, de densité de courant ou de pertes, dès que nous imposons le courant dans les conducteurs. Or c'est ce que nous réalisons pour déterminer les propriétés équivalentes.

Avec un grand nombre de cellules sur lesquelles nous appliquons l'induction macroscopique $B$, nous pouvons traduire les variations locales par l'intermédiaire d'un champ périodique $a_{p}$, qui, suivant [18], se met sous la forme :

$\mathrm{a}=\mathrm{B} \wedge \mathrm{x}+\mathrm{a}_{\mathrm{p}}$

Le champ électrique e:

$\mathbf{e}=-\mathrm{j \omega} \mathbf{a}-\operatorname{gradV}$

est exprimé à partir de $\mathrm{a}_{\mathrm{p}}$ par l' expression suivante:

$$
e=-j \omega(B \wedge x)-\operatorname{grad} V-j \omega a_{p}
$$

Le problème à résoudre consiste alors à déterminer $\mathrm{a}_{\mathrm{p}}$, grandeur périodique vérifiant les équations ci-dessous: 


$$
\begin{array}{ll}
\operatorname{rot}\left(v_{\mathrm{o}} \operatorname{rota}_{\mathrm{p}}\right)=0 & \begin{array}{l}
\text { dans l'air } \\
\text { dans le conducteur }
\end{array} \\
\operatorname{rot}\left(v_{\mathrm{o}} \operatorname{rota}_{\mathrm{p}}\right)+\mathrm{j} \omega \sigma \mathrm{a}_{\mathrm{p}}=-\sigma \operatorname{gradV}-\mathrm{j} \omega \sigma(\mathrm{B} \wedge \mathrm{x}) & \\
\mathrm{I}=\int_{\text {cond }}-\mathrm{j} \omega \sigma \mathrm{a}_{\mathrm{p}}-\sigma \operatorname{gradV}-\mathrm{j} \omega \sigma(\mathrm{B} \wedge \mathrm{x}) \mathrm{d} \Omega &
\end{array}
$$

La connaissance de $a_{p}$ et gradV après résolution permet de calculer a et e avec les expressions définies précédemment. Les grandeurs locales $b$, h et j peuvent être obtenus sans difficulté à partir de a et des lois de comportement locales.

Le système d'équations est résolu suivant deux conditions :

- en imposant une valeur de B non nulle et une valeur de courant I nulle, et ceci suivant les deux directions principales de la cellule, afin de déterminer le tenseur de reluctivité équivalent,

- en imposant $\mathrm{B}=0$ avec une valeur de courant I non nulle pour déterminer la résistivité équivalente.

Les grandeurs équivalentes complexes peuvent alors être déterminées à l'aide des expressions [14] et [15]. La résolution par éléments finis à l'échelle du dispositif est conduite en utilisant ces dernières, avec les formulations habituelles. Les pertes sur une bobine se calculent après résolution par:

$$
\mathrm{P}=\mathrm{j} \omega \int_{\Omega} \mathrm{B}\left[v_{\mathrm{eq}}\right] \overline{\mathrm{B}} \mathrm{d} \Omega+\mathrm{R}_{\mathrm{eq}} \mathrm{I} . \overline{\mathrm{I}}
$$

avec

$$
\mathrm{R}_{\mathrm{eq}}=\rho_{\mathrm{eq}} \mathrm{n}^{2} \mathrm{~V}_{\mathrm{bob}}
$$

Dans le cas d'un couplage avec les équations de circuit la relation courant tension d'une bobine s'écrit classiquement, avec une formulation utilisant le potentiel vecteur magnétique:

$$
U=R_{\text {eq }} \cdot I+j \omega \int_{\Omega_{\text {bob }}} n \cdot A d \Omega
$$

et avec une formulation de type $t_{0}-\Phi$ (très utilisé en $3 D$ ) :

$$
U=R_{\text {eq }} \cdot I+j \omega \int_{\Omega_{\mathrm{o}}} t_{\mathrm{o}} \cdot B d \Omega
$$

où $\mathrm{t}_{\mathrm{o}}$ représente le champ source créé par la bobine lorsqu'elle est parcouru par un courant de 1 A et $\Omega_{\mathrm{o}}$ le domaine de définition de $t_{\mathrm{o}}$. 
4. 4 Exemple: homogénéisation de brins rectangulaires

La formulation précédente est générale mais n'existe pas forcément en standard dans les codes par éléments finis. Cependant, dans de nombreux cas, les symétries du problème élémentaire permettent de résoudre directement les équations locales en utilisant les formulations classiques comme c'est le cas pour le problème présenté ci-dessous. Nous sommes en présence d'un ensemble de brins conducteurs rectangulaires, régulièrement espacés. Le problème est résolu par éléments finis en deux dimensions en utilisant le potentiel vecteur a.

4.4.1 Détermination de la reluctivité magnétique équivalente

Compte tenu des symétries, nous pouvons limiter la résolution locale au domaine présenté sur la figure ci-dessous:

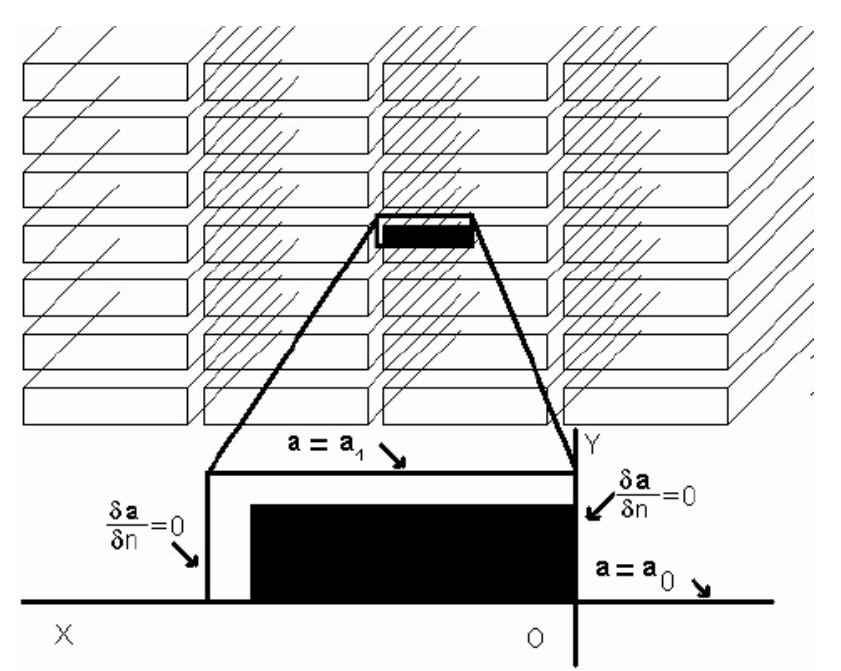

Figure 4. Cellule élémentaire d'un paquet de conducteurs rectangulaires.

Les symétries permettent, en imposant $\mathrm{a}=0$ sur le plan de symétrie de ne résoudre que l'équation de champ:

$$
\operatorname{rot}\left(v_{\mathrm{o}} \operatorname{rota}\right)+j \omega \sigma \mathbf{a}=0
$$

Avec ce choix, compte tenu de la symétrie du problème, $\langle a\rangle=0$ sur l'ensemble de la cellule élémentaire (en prenant en compte les symétries) et un courant imposé nul correspond à une tension imposée nulle car:

$$
I=\int_{\text {cond }} \mathbf{j} \mathrm{d} \Omega=\int_{\text {cond }}-\mathrm{j} \omega \sigma \mathbf{a}-\sigma \operatorname{gradV} \mathrm{d} \Omega=\sigma \mathrm{S}_{\text {cond }} \Delta \mathrm{V}
$$


La solution de l'équation de champ sur a qui exprime une tension nulle sur le conducteur, impose aussi un courant total nul, la densité de courant locale étant

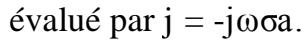

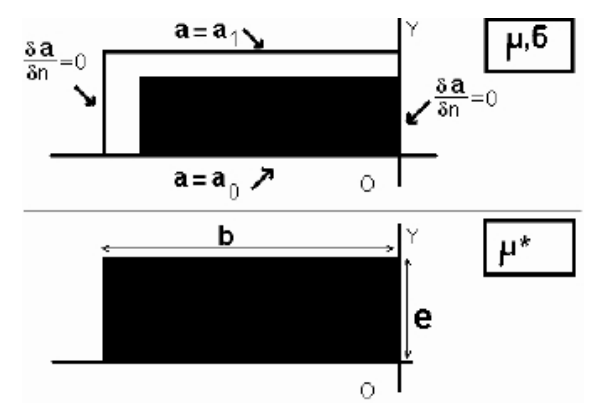

Figure 5. Passage d'un problème local à un problème homogénéisé - Conditions aux limites sur la cellule élémentaire.

Le champ macroscopique B suivant $\mathrm{x}$ est imposé par les conditions aux limites suivantes:

$$
\begin{array}{lr}
\mathbf{a}=\mathbf{a}_{\mathbf{o}}=0 & \text { sur l'axe } \mathrm{x} \\
\mathbf{a}=\mathbf{a}_{1}=\text { e.B } & \text { sur le bord supérieure de la celulle } \\
\frac{\partial \mathbf{a}}{\partial \mathbf{n}}=0 & \text { sur les bords verticaux }
\end{array}
$$

où e est la demi hauteur de la cellule.

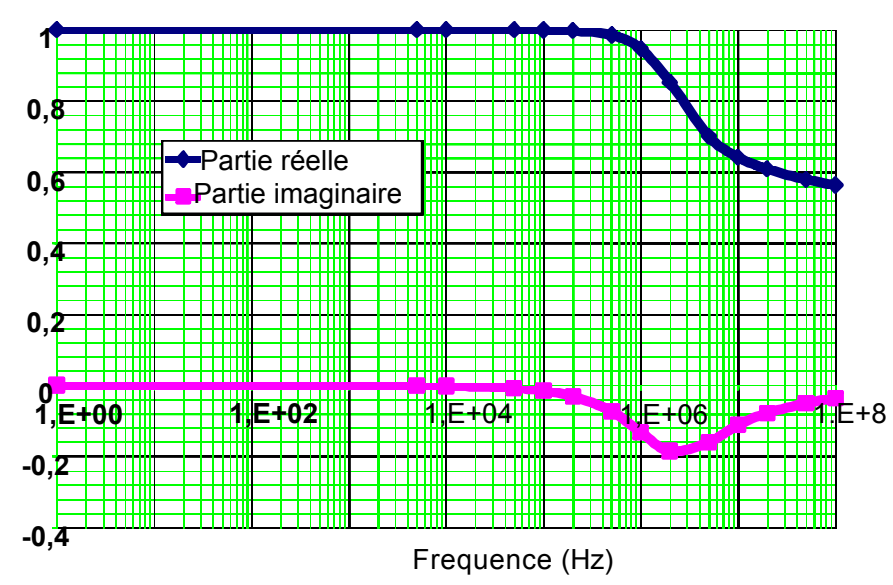

Figure 6. Perméabilité équivalente suivant y avec des brins conducteurs rectangulaires en cuivre de dimensions $0,1 \mathrm{mmx} 0,4 \mathrm{~mm}$ avec un guipage de $0,1 \mathrm{~mm}$. 
Après résolution, l'égalité des puissances actives et réactives au niveau local et macroscopique nous permet d'obtenir la reluctivité équivalente suivant $\mathrm{x}$ :

$$
v_{\mathrm{eq}_{\mathrm{xx}}}^{\prime}=\frac{1}{\mathrm{ab}} \frac{\int_{\Omega \mathrm{cel}} \mu_{0} \mathrm{~b} \overline{\mathrm{b}} \mathrm{d} \Omega}{\mathrm{B} \overline{\mathrm{B}}} \quad v_{\mathrm{eq}_{\mathrm{xx}}}^{\prime \prime}=\frac{1}{\mathrm{ab} \sigma} \frac{\int_{\Omega \mathrm{brin}} \mathrm{j} \overline{\mathrm{j}} \mathrm{d} \Omega}{\omega \overline{\mathrm{B}}}
$$

Nous procédons de façon analogue pour la direction perpendiculaire afin de déterminer $v \mathrm{q}_{\mathrm{yy}}$. La figure 6 représente un exemple de perméabilité équivalente en fonction de la fréquence pour des brins conducteurs rectangulaires.

\subsubsection{Détermination de la conductivité électrique équivalente}

Pour la conductivité électrique le problème est résolu en imposant un courant I non nul dans le conducteur (l'équation de courant associée à l'équation de champ est cette fois nécessaire) et en appliquant un champ nul sur la cellule. Ceci est obtenu en imposant $\mathrm{a}=$ cste sur le bord de la cellule. Le choix de la constante n'influence pas le résultat final, l'équation de courant permettant de retrouver la bonne répartition de courant. Cependant, puisque la variation de $\mathrm{j}$ est directement lié à celle de $\mathrm{a}$, le choix $\mathrm{a}=0$ est judicieux. Un autre choix peut conduire à des imprécisions numériques.

La résistivité équivalente est obtenue par :

$$
\rho_{\text {eq }}^{\prime}=-\frac{1}{a b} \frac{\int_{\Omega \text { cond }} \frac{1}{\sigma} \bar{j} \bar{j} d \Omega}{J \bar{J}} \quad \rho_{\text {eq }}^{\prime \prime}=\frac{1}{a b} \frac{j \omega \int_{\Omega \text { cel }} \mu_{0} b \bar{b} d \Omega}{J \bar{J}}
$$

\section{5 Validation sur un exemple bidimensionnel simple}

Nous présentons l'exemple d'une bobine conductrice composée de 36 filaments, parcourue par un courant imposé (Fig. 7) et entourée par un circuit magnétique. Nous calculons et comparons les puissances dissipées obtenues dans la bobine en utilisant ou non la technique d'homogénéisation. Nous avons maillé très finement le problème sans homogénéisation (4 éléments du second ordre dans l'épaisseur de peau), ce qui permet de définir la solution obtenue avec cette méthode comme étant la référence de notre calcul.

Les résultats, obtenus pour une fréquence définissant une épaisseur de peau égale au $1 / 3$ du coté d'un conducteur, sont résumés dans le tableau ci-après, où les pertes supplémentaires sont directement exprimées par rapport aux pertes électrocinétiques $\mathrm{P}_{\mathrm{o}}$. Nous appelons pertes propres les pertes obtenues à partir de la conductivité électrique équivalente et pertes par proximité celles obtenues à partir de la perméabilité magnétique équivalente (notons qu'en pratique il n'y a pas de champ extérieur, et que ces pertes sont globalement les pertes propres de la bobine dans son environnement). 


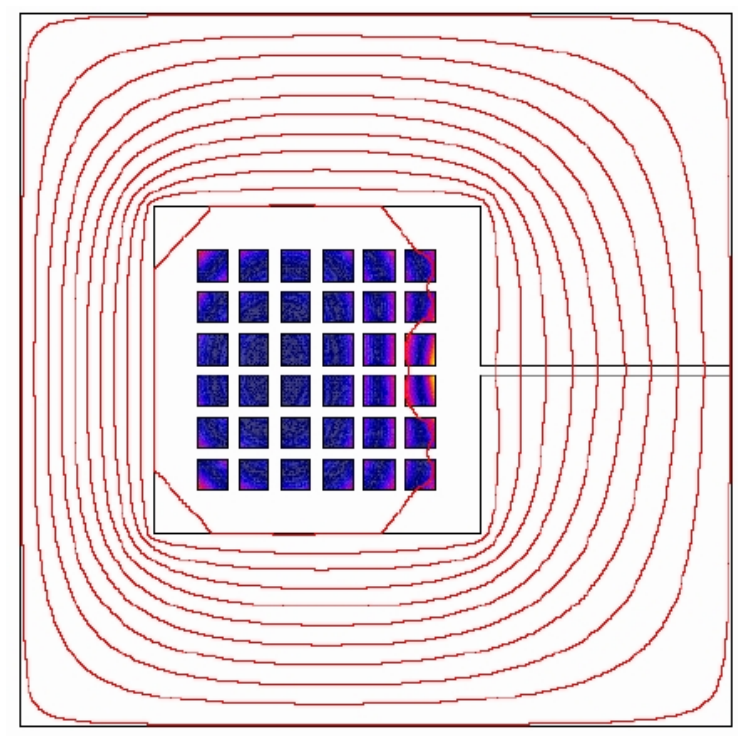

Figure 7 : Problème test bidimensionnel. Tracé des lignes de champ et des densités de courant dans chaque conducteur (dégradés).

\begin{tabular}{||c|c|c|c|c|c|c||}
\hline \multicolumn{2}{|c|}{} & \multicolumn{2}{|c|}{$\begin{array}{r}\text { Résultats obtenus en utilisant les } \\
\text { propriétés équivalentes }\end{array}$} & $\begin{array}{c}\text { Résultat } \\
\text { référence }\end{array}$ & Ecart \\
\cline { 2 - 7 } & $\mathrm{P}_{\text {propres }} / \mathrm{P}_{\mathrm{o}}$ & $\mathrm{P}_{\text {proximité }} / \mathrm{P}_{\mathrm{o}}$ & $\mathrm{P}_{\text {tot }} / \mathrm{P}_{\mathrm{o}}$ & $\mathrm{P}_{\text {tot }} / \mathrm{P}_{\mathrm{o}}$ & \\
\hline \multirow{2}{*}{$\begin{array}{l}\mu_{\mathrm{r}} \text { circuit } \\
\text { magnétique }\end{array}$} & 1 & 1,22 & 8,98 & 10,11 & 10,01 & $1 \%$ \\
\cline { 2 - 7 } & 100 & 1,22 & 12,63 & 13,90 & 13,51 & $3 \%$ \\
\hline
\end{tabular}

Tableau 1 : Validation du calcul des pertes supplémentaires, cas bidimensionnel.

Les résultats obtenus sont satisfaisants au regard du relatif petit nombre de conducteurs. Ces résultats amènent deux commentaires :

- Le calcul des propriétés équivalentes a été mené en considérant que l'induction (respectivement la densité de courant) macroscopique était uniforme sur le 
conducteur. En pratique si le nombre de conducteurs n'est pas suffisant important, l'induction magnétique macroscopique peut varier de façon significative sur un conducteur élémentaire (le flux entrant par un coté n'est pas égal au flux sortant du coté opposé). Les conséquences directes en sont une erreur sur le calcul des pertes par proximité, qui dans notre cas sont surévaluées.

- Le rapport pertes propres/pertes par proximité tend rapidement vers zéro lorsque le nombre de conducteurs devient important. Cela s'explique par le fait que les pertes par proximité (créées par la bobine sur elle-même) sont proportionnelles à $(\mathrm{nI})^{2}$ alors que les pertes électriques sont proportionnelles à $\mathrm{I}^{2}$.

\section{6 Validation des pertes par proximité sur un exemple 3D}

Nous proposons dans cette partie le calcul des pertes dans le secondaire d'un transformateur test (Figure 8) en 3 dimensions. L'étude est menée avec le secondaire en circuit ouvert, où nous cherchons à déterminer les pertes par proximité (il n'y a pas de pertes propres puisque le courant traversant les conducteurs est nul). L'enroulement est composé de deux couches de cinq brins conducteurs et la fréquence de fonctionnement peut varier de $50 \mathrm{kHz}$ à $500 \mathrm{kHz}$. Nous allons résoudre ce problème suivant deux techniques: en discrétisant les conducteurs et en utilisant la méthode d'homogénéisation. En ce qui concerne l'homogénéisation, nous avons utilisé les résultats obtenus en $2 \mathrm{D}$ pour la perméabilité complexe dans le plan perpendiculaire aux conducteurs (paragraphe 4.4) et une perméabilité égale à $\mu_{\mathrm{o}}$ dans la troisième dimension. Cette hypothèse est justifiée par le fait que le champ magnétique est principalement dans un plan perpendiculaire au conducteur.

La discrétisation du problème en éléments finis pour une résolution standard (calcul des courants de Foucault dans chaque brin) engendre un maillage de 750.000 mailles. Les calculs ont été réalisés avec le logiciel Flux®.

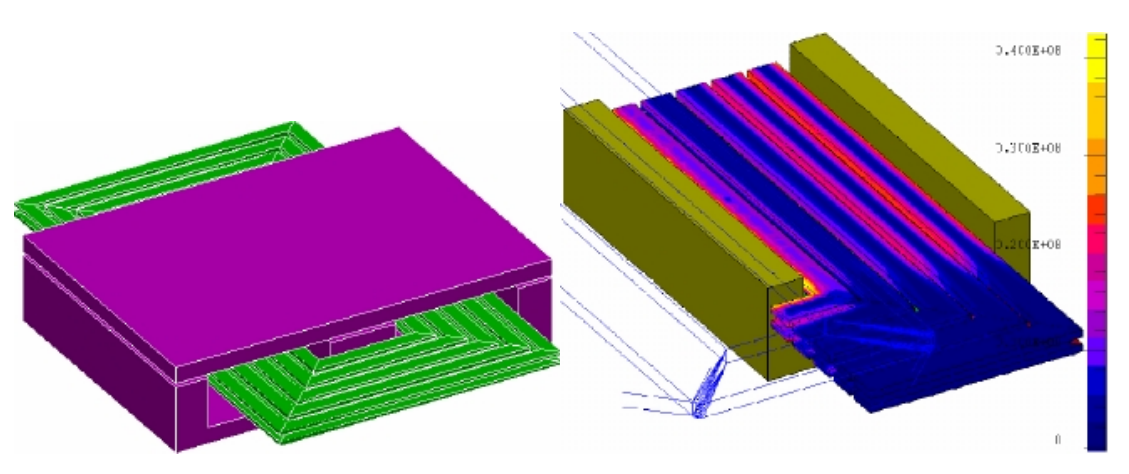


Figure 8. Problème test tridimensionnel Figure 9. Densité de courant dans les conducteurs secondaires.

Le tableau 2 compare les pertes obtenues dans le secondaire avec une résolution standard (formulation $\mathrm{T}-\mathrm{T}_{0}-\phi$, inconnues vectorielles dans les conducteurs) et ceux obtenus avec un modèle de perméabilité équivalente (formulation $\mathrm{T}_{0}-\phi$, inconnues scalaires) pour une fréquence de $500 \mathrm{kHz}$. On notera le faible temps de calcul pour le second cas, en raison d'un maillage moins dense (300.000 mailles) et de l'absence d'inconnues vectorielles.

\begin{tabular}{||c|c|c|c||}
\hline \multirow{2}{*}{} & \multicolumn{2}{|c||}{ Puissance } & \multirow{2}{*|}{ Temps CPU } \\
\cline { 2 - 3 } & Active & Réactive & \\
\hline $\begin{array}{c}\text { Résolution standard } \\
\text { (inconnues } \\
\text { vectorielles) }\end{array}$ & $1.505 \mathrm{~mW}$ & $7.07 \mathrm{mVAr}$ & 12 heures \\
\hline $\begin{array}{c}\text { Perméabilité } \\
\text { équivalente }\end{array}$ & $1.664 \mathrm{~mW}$ & $7.11 \mathrm{mVAr}$ & 90 secondes \\
\hline
\end{tabular}

Tableau 2 : Comparaison des puissances sur le secondaire en court-circuit du transformateur test 3D à 500kHz.

Malgré un nombre limité de conducteurs, lié au fait que la taille des problèmes devient rapidement très importante lorsque nous résolvons en discrétisant les brins conducteurs en $3 \mathrm{D}$, les résultats obtenus sont très satisfaisants. Là encore nous pouvons noter une légère surestimation des pertes. On peut espérer de meilleurs résultats avec un nombre de conducteurs plus important, ce qui est le cas de nombreux dispositifs en pratique.

4. 7 Orthogonalité des pertes

Dans ce qui précède nous avons supposé que les lois de comportement macroscopiques s'établissaient sous la forme:

$$
\left\{\begin{array}{l}
<\mathbf{H}> \\
<\mathbf{E}>
\end{array}\right\}=\left[\begin{array}{cc}
{\left[v_{\mathrm{eq}}\right]} & 0 \\
0 & \rho_{\mathrm{eq}}
\end{array}\right]\left\{\begin{array}{l}
\mathbf{B} \\
\mathbf{J}
\end{array}\right\}
$$

$\mathrm{H}$ et $\mathbf{J}$ d'une part et entre $\mathrm{E}$ et $\mathrm{B}$ sont couplés à travers les équations de Maxwell à l'échelle macroscopique. Cependant, comme nous avons pris en compte le couplage de ces équations à l'échelle locale et il semble légitime de penser qu'il puisse en résulter un couplage des lois de comportement à l'échelle macroscopique. 
Plaçons nous dans le cas où nous imposons un champ magnétique non nul et un courant nul. Si, après résolution du problème local sur la cellule élémentaire, la valeur moyenne macroscopique locale $\langle\mathrm{E}\rangle$ est différente de zéro, nous avons un couplage entre E et B (terme bas gauche de la matrice ci-dessus). Puisque nous imposons un courant I nul, nous écrivons :

$$
\mathbf{I}=\langle\mathbf{j}>=\lambda<\sigma \mathbf{e}>=0
$$

et par conséquent, avec une conductivité $\sigma$ constante et uniforme, nous obtenons pour E :

$$
<\mathbf{E}>=<\mathbf{e}>-<\mathbf{e}_{\mu}>=-<\mathbf{e}_{\mu}>
$$

Examinons le cas du conducteur rectangulaire (ou cylindrique) soumis à un champ magnétique suivant $\mathrm{x}$ (respectivement $\mathrm{y}$ ), en ayant imposé un courant total nul. En raison des symétries, nous pouvons facilement montrer que dans ce cas $\left\langle\mathrm{e}_{\mu}\right\rangle=0$ et que nous n'avons donc pas de dépendance entre $\mathrm{E}$ et $\mathrm{B}$. Concrètement, cela s'explique par le fait que sous l'action du champ magnétique les boucles de champ $b_{\mu}$ sont symétriques et opposées et n'engendrent donc pas de champ électrique.

De façon plus générale cette orthogonalité est directement liée au fait que nous supposons les grandeurs macroscopique $\mathrm{B}$ et $\mathbf{J}$ uniforme sur la cellule élémentaire. La dépendance de $\mathrm{E}$ en fonction de $\mathrm{B}$ peut alors s'exprimer localement à partir la loi de Maxwell-Faraday [11] suivant :

$$
E=-j \omega B \wedge x+E_{o}
$$

où $\mathrm{E}_{\mathrm{o}}$ représente le champ électrique au centre de la cellule si nous choisissons l'origine de $\mathrm{x}$ au centre de la cellule. Ce champ est le champ macroscopique obtenu avec $\mathrm{B}=0$ sur la cellule. Par conséquent le champ macroscopique local s'écrit:

$E=-j \omega B \wedge x+\rho_{\text {eq }} J$

qui implique que $\langle\mathrm{E}\rangle=0$ si $\mathrm{J}=0$. De la même façon nous pouvons établir qu'il n'y a pas dépendance entre $\mathrm{H}$ et $\mathrm{J}$ au niveau de la loi macroscopique. En pratique, l'absence de terme de couplage traduit une orthogonalité des pertes "magnétiques" et "électriques" à l'échelle où nous définissons les propriétés macroscopiques.

\section{Conclusion}

Nous avons montré comment traduire les pertes sur les brins conducteurs d'une bobine par des propriétés macroscopiques équivalentes. Dans le cas des régimes harmoniques, les perméabilités et résistivités complexes obtenues avec une méthode d'homogénéisation numérique permettent de modéliser efficacement les pertes dans les systèmes à grand nombre de conducteurs (bobines). Elles permettent la résolution du problème électromagnétique par la méthode des éléments finis, en 
tenant compte des pertes locales dans les brins conducteurs, sans nécessiter un maillage de ces derniers. Au final, la technique rend possible une modélisation précise des dispositifs sans nécessiter des temps de calcul prohibitifs.

Nous avons appliqué notre démarche au cas des bobines filaires. Cette technique peut être étendue sans difficulté aux dispositifs comprenant des fils de Litz. D'une façon plus générale, la méthodologie proposée peut s'appliquer sur tous les dispositifs où l'homogénéisation est envisageable, comme lorsque que nous sommes en présence de matériaux composite. Il est alors possible, avec la même démarche que celle que nous avons proposé pour les brins conducteurs, de résoudre un problème élémentaire 3D comprenant des milieux conducteurs et/ou perméables et réinjecter les propriétés équivalentes dans le problème macroscopique.

Enfin, des travaux ultérieurs devront permettre de pouvoir déterminer des propriétés équivalentes lorsque nous sommes en présence de propriétés locales non linéaires et dans des régimes non sinusoïdaux.

\section{Bibliographie}

Ferreira J. A., «Improved analytical modeling of conductive losses in magnetic components», IEEE Transactions on Magnetics, Vol. 30, N 1, January 1994, 127-131.

Flux ${ }^{\circledR}$, Cedrat - www.cedrat.com

Gyselinck J., Dular P., "Frequency-domain homogenization of bundles of wires in 2-D magnetodynamic FE calculations, IEEE Transactions on Magnetics, Vol. 41, Issue 5, May 2005, 1416-1419.

Matagne E ., «Modélisation magnétique macroscopique des faisceaux de conducteurs », J. Phys. III France, 3 (1993). 509-517.

Matagne E ., « Macroscopic electric characterization of bundles of conductors », IEEE Transactions on Magnetics, Vol 31, №3, May 1995, 1464-1467.

Moreau O., Popiel L., Pages J. L., «Proximity Losses computation with a 2D Complex Permeability Modelling », IEEE Trans. Magnetics, vol 34, No. 5, pp. 3616-3619, September 1998.

Nan X., Sullivan R.S., «Simplified high-accuracy calculation of eddy-current loss in roundWire Winding", Proceedings of $35^{\text {th }}$ Annual IEEE Power Electronics Specialists Conference, Aachen, Germany, 2004. »

Phung A-T., Meunier G., Chadebec O., Margueron X., Keradec J.-P., « High frequency Proximity Losses Determination for Rectangular Cross Section Conductors », IEEE Transactions on Magetics, Vol. 43, N²4, April 2007, 1213-1216.

Robert F., "Modélisation et simulation de transformateurs pour alimentations à découpage", Thèse de Docteur en Sciences Appliquées, Université Libre de Bruxelles, Août 1999

Stoll, Richard L., «The analysis of eddy current », Oxford, Clarendon Press, 1974. 